\title{
Avaliação Empírica da Proposta Interdisciplinar de Uso dos Processadores BIP
}

\author{
Paulo V. Vieira, Cesar A. Zeferino, André L. A. Raabe \\ Mestrado Acadêmico em Computação Aplicada \\ Universidade do Vale do Itajaí (UNIVALI) - Itajaí, SC - Brasil \\ \{paulovieira, zeferino, raabe\} @univali.br
}

\begin{abstract}
Resumo. A disciplina de Arquitetura e Organização de Computadores é essencial para a formação dos alunos de graduação na área de Computação, por oferecer subsídios para a redução da abstração e para um melhor entendimento sobre o funcionamento do computador. Nesse sentido, uma família de processadores e um ambiente integrado foram desenvolvidos e vêm sendo utilizados em diferentes disciplinas, promovendo a interdisciplinaridade. Este artigo avalia os beneficios dessa proposta em disciplinas de Arquitetura e Organização de Computadores e Compiladores. A pesquisa segue um delineamento quase-experimental, com a aplicação de experimentos a quatro turmas de alunos. Os resultados demonstram que os alunos da disciplina Compiladores perceberam melhor a influência da arquitetura do processador na etapa de geração de código e apresentarem uma melhoria na aprendizagem. Os alunos de Arquitetura e Organização de Computadores não apresentaram melhoria significativa na aprendizagem.
\end{abstract}

\begin{abstract}
The discipline of Computer Architecture and Organization is essential to the formation of undergraduates in the Computer Science area. It provides the necessary subsidies to reduce abstraction and helps to understand the internal workings of the computer. In this sense, a family of processors and an integrated development environment were developed. These resources have been used in different disciplines, promoting the interdisciplinary integration. This work aims at empirically assessing the benefits of this proposal in the disciplines of Computer Architecture and Organization, and Compilers. The research follows a quasi-experimental design. Experiments were conducted with four groups of students. The results showed that Compiler students perceived better the influence of processor architecture on code generation, and showed an improvement in learning. The students of Computer Architecture and Organization showed no significant improvement in learning.
\end{abstract}

\section{Introdução}

Aprender a programar é um processo difícil e exigente para a maioria dos alunos da área de Computação, especialmente àqueles de disciplinas introdutórias de programação. Renumol, Janakiram e Jayaprakash (2010) acreditam que essa dificuldade é agravada pelo alto nível de abstração envolvido nos conceitos iniciais de programação. Nesse sentido, diversas soluções têm sido adotadas para reduzir a abstração, motivar os estudantes e melhorar o processo de ensino e aprendizagem em programação. 
As organizações ACM (Association for Computing Machinery) e IEEE (Institute of Electrical and Electronics Engineers) salientam a importância do ensino de arquitetura e organização de computadores [Cassel et al. 2008]. De acordo com Cassel (2008), os estudantes de Ciência da Computação devem compreender os componentes funcionais de um computador, suas características, interações e desempenho. Zeferino et al. (2012) acreditam que o estudo da arquitetura do computador possibilita estabelecer as relações dos conceitos abstratos de programação com aspectos concretos do hardware. Khalife (2006) propõe a utilização de um modelo simplificado de computador, possibilitando aos alunos iniciantes compreender de forma adequada os conceitos de programação.

Nesse contexto, Morandi, Raabe e Zeferino (2006) especificaram uma família de processadores denominada BIP (Basic Instruction-set Processor). Esses processadores apresentam um conjunto reduzido de instruções e podem ser utilizados para exemplificar conceitos introdutórios de computação. Vieira, Raabe e Zeferino (2010) desenvolveram um ambiente de desenvolvimento integrado, denominado Bipide, o qual facilita a adoção dos processadores BIP em sala de aula.

De acordo com Zeferino et al. (2012), as iniciativas de criação e adoção dos processadores BIP e do Bipide em sala de aula permitiu que fosse dado um enfoque interdisciplinar aos conceitos de arquitetura e organização de computadores, vislumbrando seu desdobramento em diferentes disciplinas. Avaliações demonstraram os benefícios dessa proposta para a redução da abstração envolvida nos conceitos de uma disciplina de Algoritmos e Programação [Vieira, Raabe e Zeferino 2010]. No entanto, nenhuma avaliação foi realizada em outras disciplinas do curso.

Considerando o exposto, esta pesquisa avalia empiricamente o efeito da proposta interdisciplinar baseada nos processadores BIP sobre a aprendizagem dos alunos das disciplinas Arquitetura e Organização de Computadores (AOC) e Compiladores. A pesquisa foi abordada de forma quantitativa, seguindo um delineamento quaseexperimental com grupos de controle não-equivalentes. Além disso, foi conduzida uma análise qualitativa na disciplina Compiladores, com a realização de um grupo focal.

\section{Processadores BIP}

Nas fases iniciais de um curso de graduação, os processadores utilizados devem priorizar aspectos didáticos que permitam estabelecer a relação entre as abstrações lógicas da programação e sua representação em hardware [Khalife 2006]. Nota-se, entretanto, que os modelos de processadores utilizados em disciplinas introdutórias são abstratos demais e não permitem estabelecer essas relações. Uma alternativa seria utilizar processadores mais detalhados, porém, esses processadores são demasiadamente complexos, e poucos são os livros texto que propiciam a integração entre a arquitetura do processador e a programação em alto nível [Zeferino et al. 2012].

Discussões continuadas entre professores de diferentes áreas do curso de Ciência da Computação na UNIVALI levaram à concepção de uma família de processadores com arquitetura simplificada [Morandi, Raabe e Zeferino 2006]. Essa família é composta por uma série de processadores denominados BIP (Basic Instruction-set Processor), os quais foram desenvolvidos com níveis incrementais de complexidade, vislumbrando a representação de conceitos em diferentes disciplinas da área de Computação. Para Zeferino et al. (2012), essa iniciativa demonstra o enfoque interdisciplinar dos 
processadores BIP, sendo esse o seu principal diferencial em relação a processadores similares.

\section{Ambiente Integrado Bipide}

Em cursos da área de Computação, a utilização de simulação por meio de diferentes técnicas de visualização colabora para o desenvolvimento de um modelo mental preciso sobre o processo de execução de programas [Djordjevic et al. 2000]. Conhecendo esses benefícios, Vieira, Raabe e Zeferino (2010) desenvolveram o ambiente de desenvolvimento integrado Bipide, o qual relaciona conceitos de programação em alto nível com o hardware dos processadores BIP. Esse ambiente permite a criação e execução de algoritmos e exibe o estado dos componentes da organização do processador através de animações que ilustram o funcionamento interno do mesmo.

Zeferino et al. (2012) relatam a utilização do Bipide em diferentes disciplinas de um curso de Ciência da Computação. Vieira, Raabe e Zeferino (2010) demonstraram que com a utilização do Bipide, os alunos de uma disciplina de programação introdutória puderam consolidar os conceitos estudados na disciplina e apresentaram uma melhoria de desempenho da ordem de 1,928 pontos na média com $99 \%$ de confiança.

\section{Trabalhos Relacionados}

Foram considerados como trabalhos relacionados a esta pesquisa, àqueles que tratam de avaliações de propostas interdisciplinares na área de Computação. Para a seleção dos trabalhos, foi realizado um mapeamento sistemático a partir de um protocolo de busca, composto por diversos termos pertinentes ao tema pesquisado.

A pesquisa foi realizada em quatro bases de dados: (i) IEEE Xplore; (ii) ScienceDirect; (iii) ACM Digital Library; e (iv) SpringerLink. Os trabalhos foram selecionados por meio da leitura de seus resumos, onde foram excluídos aqueles que não atendiam aos critérios de seleção estabelecidos. A Tabela 1 apresenta um resumo sobre a busca realizada, informando a quantidade de trabalhos selecionados para avaliação.

Tabela 1. Resumo da busca de trabalhos relacionados

\begin{tabular}{ccccc}
\hline Base de Dados & Total de trabalhos & Trabalhos selecionados & Trabalhos eliminados & Trabalhos analisados \\
\hline IEEE Xplore & 153 & 37 & 149 & 4 \\
ScienceDirect & 99 & 21 & 78 & 21 \\
ACM Digital Library & 100 & 36 & 91 & 9 \\
SpringerLink & 67 & 9 & 58 & 9 \\
\hline Total & 419 & 103 & 376 & 43 \\
\hline
\end{tabular}

Após leitura e análise dos trabalhos selecionados pôde-se perceber que a maioria não apresentava detalhes suficientes sobre a pesquisa. Alguns não apresentavam o delineamento do experimento e/ou os resultados obtidos. Outros trabalhos sequer descreviam os métodos didáticos ou alterações curriculares realizadas.

Cerca de $40 \%$ das pesquisas utilizaram de grupos de controle, nas quais se aplicou uma intervenção e, em seguida, mediu-se a aprendizagem através de um questionário. Percebeu-se um grande número de pesquisas que utilizam estratégias de pesquisa qualitativa (44\%), com a aplicação de questionários para coleta de dados, como se nota na Figura 1. Em relação ao nível de ensino, a maioria das pesquisas $(60,5 \%)$ foi realizada em cursos de graduação e envolveram apenas um curso ou disciplina. 
Estratégias de Pesquisa

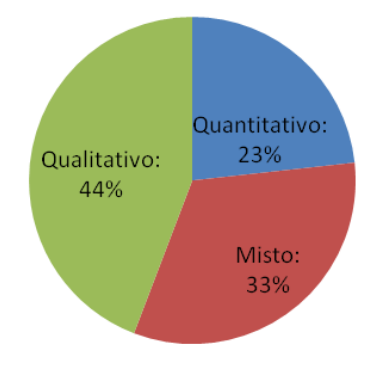

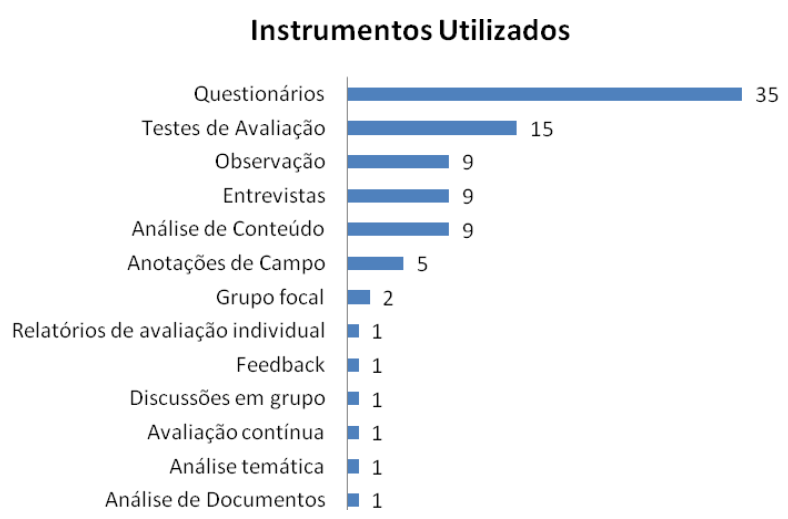

Figura 1. Estratégias de Pesquisa e Instrumentos utilizados para coleta de dados

\section{Pesquisas Empíricas em Educação}

$\mathrm{Na}$ área da Educação, as pesquisas realizadas têm como objetivo principal o ensino. No entanto, é necessário considerar o que se espera que seja aprendido. Dessa forma, pode-se dizer que o fenômeno de interesse da pesquisa em ensino envolve não só o ensino, em si, mas também a aprendizagem, a avaliação e o currículo.

Para saber se houve aprendizagem é necessário avaliá-la. A avaliação da aprendizagem pode prover evidências não só sobre o que foi aprendido, mas também sobre até que ponto o ensino foi responsável por isso. Nesse aspecto, são vários os eventos que contribuem para se alcançar esse objetivo, tais como uma aula expositiva, um novo currículo, comportamentos do professor, entre outros.

\section{Metodologia da Pesquisa}

Este trabalho tem como hipótese geral que a apresentação de conceitos sobre o funcionamento do computador, utilizando um modelo simplificado representado pela arquitetura dos processadores BIP, e o subsequente uso da ferramenta Bipide influenciam positivamente a aprendizagem dos alunos de cursos de Ciência da Computação. Buscouse comprovar essa hipótese através de uma avaliação empírica que busca responder à seguinte questão: “A utilização da família de processadores BIP influencia positivamente na aprendizagem dos alunos nas disciplinas de Arquitetura e Organização de Computadores e de Compiladores?".

O problema central da pesquisa foi abordado de maneira quantitativa, seguindo um modelo quase-experimental conhecido como "Grupo de Controle não Equivalente" [Campbell e Stanley 1979]. Esse delineamento implica na aplicação de instrumentos de avaliação a um grupo de controle e a um grupo experimental na forma de pré-teste e pósteste. As seguintes hipóteses foram verificadas:

- $H_{0}$ : A média dos alunos do grupo experimental é menor ou igual à média do grupo de controle.

- $H_{1}$ : A média dos alunos do grupo experimental é maior que a média do grupo de controle.

A pesquisa envolve disciplinas que possuem enfoques diferentes, portanto, hipóteses distintas foram definidas para cada uma das disciplinas: 
- $H_{2}$ : Os estudantes do grupo experimental perceberam mais claramente a distinção entre a arquitetura e a organização do computador.

- $H_{3}$ : Os estudantes do grupo experimental perceberam melhor a influência da arquitetura do computador nas diferentes etapas de construção do compilador do que os alunos do grupo de controle.

As amostras utilizadas na pesquisa foram constituídas por alunos das disciplinas AOC ( $3^{\circ}$ período) e Compiladores ( $7^{\circ}$ período). Os alunos dessas disciplinas no segundo semestre de 2011 foram tratados como grupo de controle; já os alunos matriculados no primeiro semestre de 2012 constituíram o grupo experimental, submetidos à intervenção pedagógica proposta nesta pesquisa.

\section{Intervenções Realizadas com as Turmas de Alunos}

$\mathrm{Na}$ disciplina AOC adotava-se o processador MIPS como arquitetura de referência. Era percebido pelo professor da disciplina que a arquitetura do MIPS, por não ter sido especificada visando o aprendizado, mas sim a facilidade de implementação do processador e o seu desempenho, dificultava o entendimento inicial dos conceitos de arquitetura e de organização do computador. Dessa forma, a intervenção na disciplina consistiu na inserção dos processadores BIP como referência para ilustrar os conceitos iniciais de arquitetura e organização de computadores. Além disso, os processadores BIP também foram utilizados para traçar um comparativo de suas características arquiteturais com as características do processador MIPS, o que permitiu um aumento incremental da complexidade dos temas abordados na disciplina ao longo do semestre.

As estratégias utilizadas na disciplina AOC compreenderam a elaboração de um plano de aula, incluindo aulas teóricas e práticas. As aulas teóricas foram realizadas em sala de aula, com a utilização de uma apresentação em PowerPoint e, posteriormente foram realizadas aulas práticas em laboratórios de informática, onde os alunos puderam exercitar o desenvolvimento de programas em Assembly e observar a organização dos processadores BIP por meio do simulador Bipide.

A abordagem adotada na disciplina Compiladores utilizava uma linguagem intermediária baseada em uma máquina virtual de pilha, a qual abstraia a arquitetura do computador. A intervenção realizada, portanto, buscou demonstrar todas as fases de construção de um compilador, na qual se define a arquitetura de uma linguagem alvo para então se definirem os aspectos sintáticos e léxicos da linguagem de alto nível.

Para cumprir esse objetivo foi realizada uma aula teórica sobre lógica de programação Assembly e sobre o conjunto de instruções dos processadores BIP e realizaram exercícios de conversão de código em alto nível para código Assembly. Em seguida, os alunos assistiram uma apresentação sobre a ferramenta Bipide e foram convidados a desenvolver programas em Assembly no simulador, quando puderam comparar os códigos em alto nível com o código Assembly gerado pela ferramenta. Posteriormente foram conduzidas apresentações em sala de aula enfatizando a criação de ações semânticas e foram dadas orientações para o desenvolvimento de trabalhos individuais, nos quais os alunos deveriam construir um compilador capaz de traduzir uma linguagem de alto nível qualquer em código Assembly para os processadores BIP. 
Os planos de aula e materiais didáticos desenvolvidos foram disponibilizados em website [Bipide 2013].

\section{Instrumentos de Avaliação e Coleta de Dados}

O desempenho dos alunos nos tópicos de interesse desta pesquisa foi mensurado antes (pré-teste) e depois (pós-teste) da intervenção realizada por meio de instrumentos de avaliação específicos para cada uma das disciplinas.

$\mathrm{Na}$ disciplina AOC foram utilizados dois instrumentos distintos, um para o préteste e outro para o pós-teste. Esses instrumentos foram aplicados aos grupos de controle e experimental na primeira e na última semana do semestre letivo, respectivamente. $\mathrm{O}$ instrumento utilizado no pré-teste contém oito questões de múltipla escolha e o instrumento utilizado no pós-teste contém sete questões de resposta múltipla, ambos envolvendo tópicos sobre arquitetura e organização de computadores.

$\mathrm{Na}$ disciplina Compiladores foi utilizado o mesmo instrumento para o pré e pósteste realizados nos grupos de controle e experimental. $\mathrm{O}$ instrumento contém oito questões de múltipla escolha envolvendo tópicos sobre compiladores de interesse a esta pesquisa. A aplicação do pré-teste ocorreu em torno da décima semana do semestre letivo, enquanto o pós-teste foi aplicado aos grupos na penúltima semana.

\section{Resultados na Disciplina AOC}

O teste de Mann-Whitney foi utilizado para avaliar se as amostras pertenciam a populações com a mesma distribuição. Esse teste não apresentou significância estatística $(W=182 ; p=0,722)$ e, portanto, se aceita a hipótese de que os grupos apresentam equivalência pré-experimental de amostragem. Para testar a normalidade das amostras foi utilizado o teste Shapiro-Wilk, o qual demonstrou que o grupo de controle ( $W=0,843 ; p$ $=0,023)$ e o grupo experimental $(W=0,929 ; p=0,047)$ não seguiam uma distribuição normal. Esse resultado levou a realização de uma análise de covariância (ANCOVA) para comparação entre as médias dos grupos, onde foram utilizadas como covariáveis as pontuações obtidas no pré-teste.

Em termos gerais, a ANCOVA aplica estimadores da regressão linear entre a variável resposta e a covariável a fim de tornar possível calcular a diferença entre as médias iniciais e as médias finais, já ajustadas em função da covariável [Mourão Júnior 2010]. A Tabela 2 apresenta as médias iniciais (pré-teste) e as médias ajustadas (pósteste) obtidas pelo grupo de controle e experimental.

Tabela 2. Pontuação média obtida no pré e pós-teste na disciplina AOC

\begin{tabular}{cccccc}
\hline \multirow{2}{*}{ Grupo } & \multirow{2}{*}{$n$} & Média & Desvio Padrão & Média Ajustada & $p$ \\
\cline { 3 - 6 } & & 3,923 & 0,954 & 4,145 & 0,455 \\
Controle & 13 & 4,1 & 1,348 & 3,739 & \\
Experimental & 30 & &
\end{tabular}

No pré-teste o grupo de controle apresentou pontuações médias menores que o grupo experimental. Comparando as médias iniciais e as médias finais ajustadas, verificase que a média do grupo experimental diminuiu, enquanto a do grupo de controle aumentou. Essa diferença é demonstrada no gráfico da Figura 2. 


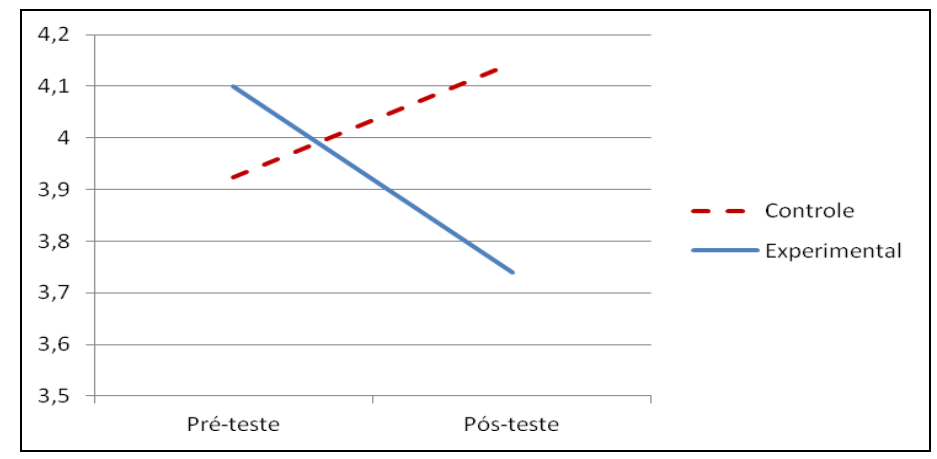

Figura 2. Gráfico da diferença entre as médias na disciplina AOC

No entanto, os resultados obtidos pela ANCOVA, apresentados na Tabela 3 indicam que não houve significância na comparação entre as médias ( $p=0,455 ; \alpha=$ $0,05)$. Portanto, não há evidência suficiente para concluir que a utilização dos processadores BIP influenciou na aprendizagem dos alunos, seja para melhor ou para pior. Dessa forma, na disciplina AOC, admite-se a hipótese nula: " $H_{0}$ : A média dos alunos do grupo experimental é menor ou igual à média do grupo de controle".

Tabela 3. Análise de Covariância utilizando escores iniciais como covariável

\begin{tabular}{cccccc}
\hline Fonte de Variação & Soma de Quadrados & Graus de Liberdade & Quadrados Médios & $F$ & $p$ \\
\hline Médias ajustadas & 1,53 & 1 & 1,53 & 0,57 & 0,455 \\
Erro ajustado & 107,41 & 40 & 2,69 & & \\
Ajuste total & 108,95 & 41 & & & \\
\hline
\end{tabular}

Para testar a segunda hipótese da pesquisa, aplicou-se o teste $\mathrm{Z}$ para verificar se houve uma melhoria na proporção de acertos em uma das questões do instrumento de avaliação. Para $\alpha=0,05$, o teste $Z$ não apresentou significância $(Z=0,297 ; p=0,766)$. Portanto, não se pode afirmar que os processadores BIP contribuíram para uma melhor distinção entre a arquitetura e organização do computador. Dessa forma, não se confirma a segunda hipótese de pesquisa: " $H_{2}$ : Os estudantes do grupo experimental perceberam mais claramente a distinção entre a arquitetura e a organização do computador".

\section{Resultados na Disciplina Compiladores}

Aplicando o teste Shapiro-Wilk verificou-se que o grupo de controle apresentou uma distribuição normal $(W=0,886 ; p=0,181)$. No entanto, o grupo experimental não seguiu a distribuição normal $(W=0,810 ; p=0,007)$, o que levou à utilização do teste nãoparamétrico de Mann-Whitney que, para $\alpha=0,05$, não apresentou significância estatística $(W=48,5 ; p=0,341)$. Dessa forma, admite-se que os grupos apresentam equivalência pré-experimental de amostragem.

Para verificar a diferença entre as médias utilizou-se a análise de covariância. $\mathrm{Na}$ avaliação inicial o grupo experimental apresentou, em média, valores ligeiramente maiores do que o grupo de controle, conforme apresentado na Tabela 4.

Tabela 4. Pontuação média obtida no pré e pós-teste na disciplina Compiladores

\begin{tabular}{cccccc}
\hline \multirow{2}{*}{ Grupo } & \multirow{2}{*}{$n$} & Média & Desvio Padrão & Média Ajustada & $p$ \\
\cline { 2 - 6 } & & 2,667 & 1,581 & 1,796 & 0,006 \\
Controle & 9 & 2,929 & 0,829 & 3,059 & \\
Experimental & 14 & &
\end{tabular}


Comparando as médias iniciais com as médias ajustadas do pós-teste, verifica-se uma modificação nos valores das médias de acertos dos alunos. Essa diferença fica evidente no gráfico ilustrado na Figura 3, onde se percebe que a média do grupo experimental aumentou, enquanto que a média do grupo de controle diminuiu.

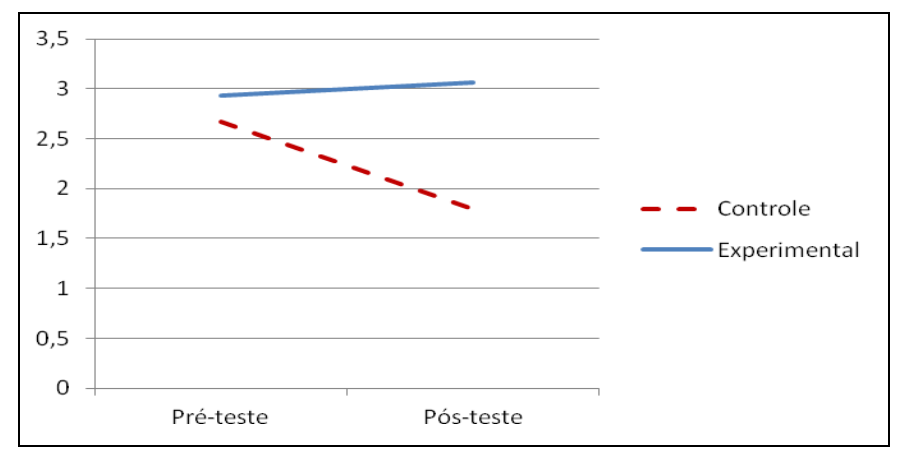

Figura 3. Gráfico da diferença entre as médias na disciplina Compiladores

Os resultados obtidos pela ANCOVA, apresentado na Tabela 5, demonstram que há evidências estatísticas sobre a diferença entre os grupos $(p=0,006)$. Portanto, pode-se afirmar que a inclusão dos processadores BIP na disciplina Compiladores produziu um efeito de melhoria na aprendizagem dos alunos, confirmando a hipótese de pesquisa: " $H_{l}$ : A média dos alunos do grupo experimental é maior que a média do grupo de controle".

Tabela 5. Análise de Covariância utilizando escores iniciais como covariável

\begin{tabular}{cccccc}
\hline Fonte de Variação & Soma de Quadrados & Graus de Liberdade & Quadrados Médios & $F$ & $p$ \\
\hline Médias ajustadas & 8,62 & 1 & 8,62 & 9,54 & 0,006 \\
Erro ajustado & 18,08 & 20 & 0,9 & & \\
Ajuste total & 26,71 & 21 & & & \\
\hline
\end{tabular}

Para testar a terceira hipótese, aplicou-se o teste $\mathrm{Z}$ em uma das questões do instrumento de avaliação do pós-teste. $O$ teste $Z$ demonstrou uma melhora significativa na proporção de acertos para $\alpha=0,05(Z=-2,66 ; p=0,008)$. A questão analisada refere-se a influencia da arquitetura do processador na fase de geração de código da construção de um compilador. Portanto, considera-se essa mais uma evidência da efetividade da proposta didática aplicada, uma vez que se comprova a terceira hipótese de pesquisa: " $H_{3}$ : Os estudantes do grupo experimental perceberam melhor a influência da arquitetura do computador nas diferentes etapas de construção do compilador do que os alunos do grupo de controle".

As descobertas obtidas na disciplina Compiladores são fortalecidas pelas percepções evidenciadas durante a realização de um grupo focal, onde os alunos expressaram que a simplicidade dos processadores BIP colaborou para a realização das atividades da disciplina. Além disso, os alunos demonstraram satisfação com os materiais didáticos disponibilizados, julgando-os suficientes para a realização das tarefas.

\section{Conclusões}

Esta pesquisa teve como objetivo principal avaliar o efeito de uma prática pedagógica interdisciplinar baseada na utilização dos processadores BIP sobre a aprendizagem dos alunos das disciplinas AOC e Compiladores. Para alcançar esse objetivo foram realizados estudos teóricos sobre os temas necessários para a realização da pesquisa e um 
mapeamento sistemático sobre trabalhos que tratam de avaliações de propostas interdisciplinares na área de Computação. Posteriormente, para mensurar a aprendizagem dos alunos foi conduzido um estudo quase-experimental, com a aplicação de pré e pósteste a grupos de controle e experimental.

A avaliação realizada na disciplina AOC, não demonstrou evidências estatísticas de que a inclusão dos processadores BIP produziu qualquer efeito na aprendizagem dos alunos, já que o grupo experimental apresentou um desempenho médio equivalente ao grupo de controle. Vale ressaltar que os processadores BIP foram utilizados na disciplina AOC como um modelo inicial de processador, não sendo alvo de estudos durante toda a disciplina. Em função disso, acredita-se que a alteração realizada no programa da disciplina não tenha sido suficiente para representar uma melhoria na aprendizagem geral dos alunos no decorrer do semestre.

$\mathrm{Na}$ disciplina Compiladores, a alteração no programa da disciplina representou uma mudança significativa, já que os processadores BIP passaram a ser o processadoralvo da disciplina. Essa mudança foi percebida nas avaliações realizadas, onde os resultados obtidos apresentaram evidências significativas que demonstram uma melhoria na aprendizagem dos alunos. Além disso, ficou evidente que a adoção do BIP facilitou a atividade de construção de um compilador, principalmente na etapa de geração de código.

Com os resultados mencionados anteriormente, pôde-se responder a questão que motivou esse estudo. As evidências encontradas mostram que:

- A utilização dos processadores BIP não produziu qualquer efeito significativo na aprendizagem dos alunos de AOC. Os alunos que aprenderam sobre os processadores BIP não progrediram e nem regrediram mais do que os que não aprenderam sobre esses processadores, pois tiveram desempenhos semelhantes.

- A utilização dos processadores BIP produziu um efeito positivo na aprendizagem dos alunos na disciplina Compiladores. Ou seja, os alunos que utilizaram os processadores BIP progrediram mais que os alunos que não utilizaram esses processadores. Além disso, esses alunos perceberam melhor a influência da arquitetura do processador na etapa de geração de código;

Os resultados obtidos nesta pesquisa são passíveis de discussão. O número reduzido de participantes nas amostras pode ter contribuído para que alguns resultados não tenham apresentado significância estatística. Além disso, em todos os grupos houve abandono de participantes, reduzindo ainda mais as amostras iniciais. A utilização de diferentes instrumentos de avaliação na disciplina AOC pode ter influenciado os resultados, pois esses instrumentos podem ter tido diferentes níveis de dificuldade. Entretanto, os mesmos instrumentos foram aplicados nas diferentes turmas e, se essa ameaça influenciou um grupo, também pode ter influenciou o outro grupo de forma semelhante.

A principal contribuição desta pesquisa foi propor uma prática pedagógica incluindo os processadores BIP nas disciplinas Compiladores e AOC. Foram disponibilizados planos de aula e materiais didáticos, os quais possibilitarão a adoção desses processadores por outros professores e instituições de ensino. Colabora para isso a disponibilidade de um website com os materiais desenvolvidos [Bipide 2013]. 
Espera-se que este trabalho sirva de referência para outras pesquisas e que os métodos e procedimentos adotados possam ser seguidos e/ou aperfeiçoados. Como trabalho futuro sugere-se a replicação dos experimentos com um número maior de alunos, o que pode evidenciar novas descobertas. Pode-se ainda aplicar novos testes estatísticos, que evidenciem aspectos não levantados nesta pesquisa.

\section{Referências}

Belli, G. (2009) "Nonexperimental Quantitative Research”, In: Lapan, S. D.; Quartaroli, M. T. Research Essentials: An Introduction to Designs and Practices. San Francisco: Jossey-Bass.

Bipide. (2013) "Ambiente de Desenvolvimento Integrado para a família de processadores BIP” http://www.bipide.com.br.

Campbell, D. T.; Stanley, J. C. (1979) "Delineamentos experimentais e quase experimentais de pesquisa”. São Paulo: EPU.

Cassel, L. et al. (2011) "Computer Science Curriculum 2008: An Interim Revision of CS 2001”. Association for Computing Machinery and IEEE Computer Society, http://www.acm.org/education/curricula/ComputerScience2008.pdf.

Djordjevic, J.; Milenkovic, A.; Grbanovic, N. (2000) "An Integrated Environment for Teaching Computer Architecture", In: IEEE Micro, v. 20, n. 3, p. 66-74.

Heath, L. (2005) "Quasi-Experiment", In: Kempf-Leonard, K. Encyclopedia of Social Measurement, v. 3. San Diego: Elsevier Science Limited, p. 255-261.

Khalife, J. T. (2006) "Threshold for the Introduction of Programming: Providing Learners with a Simple Computer Model", In: 28th International Conference on Information Technology Interfaces, Cavtat. p. 71-76.

Marczyk, G.; DeMatteo, D.; Festinger, D. (2005) "Essentials of Research Design and Methodology". Hoboken: John Wiley \& Sons.

Morandi, D.; Raabe, A. L.; Zeferino, C. A. (2006) "Processadores para ensino de conceitos básicos de arquitetura de computadores", In: Workshop de Educação em Arquitetura de Computadores. Ouro Preto, SBC, p. 17-24.

Mourão Júnior (2009) "Questões em bioestatística: o tamanho da amostra". In: Revista Interdisciplinar de Estudos Experimentais, v. 1, n. 1, p. 26-28.

Renumol, V. G.; Janakiram, D.; Jayaprakash, S. (2010) "Identification of Cognitive Processes of Effective and Ineffective Students During Computer Programming", In: ACM Transactions on Computing Education, v. 10, n. 3. New York.

Sá, J. P. M. (2007) “Applied Statistics Using SPSS, Statistica, Matlab And R2“. Porto: Springer, 2007.

Smith, E. R. (2000) "Research design", In: REIS, H. T.; Judd, C. M. Handbook of Research Methods in Social and Personality Psychology. Cambridge: Cambridge University Press, p. 17-39.

Sorva, J. (2012) "Visual Program Simulation in Introductory Programming Education". Doctoral dissertation. Department of Computer Science and Engineering. Aalto University. Espoo.

Vieira, P. V.; Raabe, A. L. A.; Zeferino, C. A. (2010) "Bipide: ambiente de desenvolvimento integrado para a arquitetura dos processadores BIP”, In: Revista Brasileira de Informática na Educação, v. 18, n. 1.

Zeferino, C. A. et al. (2012) "Um Enfoque Interdisciplinar no Ensino de Arquitetura de Computadores", In: Martins, C; Navaux P.; Azevedo, R.; Kofuji, S. Arquitetura de Computadores: educação, ensino e aprendizado. 